\title{
7. On the Logarithmic Property of the Indices of Endomorphism on a Linear Space
}

\author{
By Perla López de Cicileo and Kiyoshi Iséki \\ (Comm. by Kinjirô KunUGI, M.J.A., Jan. 13, 1964)
}

Let $E$ be a linear space, and let $u$ be an endomorphism on $E$. We denote the dimension of $u^{-1}(0)$ by $\operatorname{dim}(\operatorname{Ker}(u))$, and the codimension of $u(E)$ by $\operatorname{cod}(\operatorname{Im}(u))$. If one of these values is finite, $d(u)$ $=\operatorname{dim}(\operatorname{Ker}(u))-\operatorname{cod}(\operatorname{Im}(u))$ is called the index of $u$.

In his paper [1], M. Audin proved the logarithmic property of the indices:

THEOREM. Let $u, v$ be endomorphisms on $E$. If one of the indices of $u, v$ is finite, then

$$
d(v \circ u)=d(u)+d(v) .
$$

In this note, we shall show that the result follows from the Deprit useful lemma [2]. We only consider that $d(u), d(v)$ are finite (see M. Audin [1]).

Lemma (Deprit). Let $E, F$ and $G$ be linear spaces, and let $u(v)$ be a linear transformation from $E(F)$ to $F(G)$. Then $E$ is the direct sum of three linear spaces satisfying the following conditions:

1) $E=\operatorname{Ker}(u) \oplus E_{1} \oplus E_{2}$,

2) $\operatorname{Ker}(v \circ u)=\operatorname{Ker}(u) \oplus E_{1}$,

3) $\operatorname{Im}(v \circ u)=v\left(u\left(E_{2}\right)\right)$,

4) $E_{1}$ is isomorphic with $\operatorname{Ker}(v) \cap \operatorname{Im}(u)$,

5) $E_{2}$ is isomorphic with $\operatorname{Im}(u) / \operatorname{Ker}(v) \cap \operatorname{Im}(u)$.

Let $d(u)$ and $d(v)$ be finite indices, then, from 2) and 4), we have $\operatorname{dim}(\operatorname{Ker}(v \circ u))=\operatorname{dim}(\operatorname{Ker}(u))+\operatorname{dim} E_{1}$

$$
=\operatorname{dim}(\operatorname{Ker}(v))+\operatorname{dim}(\operatorname{Ker}(v) \cap \operatorname{Im}(u)) \text {. }
$$

On the other hand, we have an isomorphic relation:

If $E=F=G$, then

$$
F /(\operatorname{Im}(u)+\operatorname{Ker}(v)) \simeq \operatorname{Im}(v) / \operatorname{Im}(v \circ u) .
$$

Hence

$$
E /(\operatorname{Im}(u)+\operatorname{Ker}(v)) \simeq \operatorname{Im}(v) / \operatorname{Im}(v \circ u) .
$$

$$
\begin{aligned}
\operatorname{dim} & (\operatorname{Im}(v))-\operatorname{dim}(\operatorname{Im}(v \circ u)) \\
& =\operatorname{dim} E-\operatorname{dim}(\operatorname{Im}(u)+\operatorname{Ker}(v)) \\
& =\operatorname{dim} E-[\operatorname{dim}(\operatorname{Im}(u))+\operatorname{dim}(\operatorname{Ker}(v))-\operatorname{dim}(\operatorname{Im}(u) \cap \operatorname{Ker}(v))] \\
& =\operatorname{cod}(\operatorname{Im}(u))-\operatorname{dim}(\operatorname{Ker}(v))+\operatorname{dim}(\operatorname{Im}(u) \cap \operatorname{Ker}(v)) .
\end{aligned}
$$

Therefore, we have

$\operatorname{dim} E-\operatorname{dim}(\operatorname{Im}(v \circ u))=\operatorname{cod}(\operatorname{Im}(u))-\operatorname{dim}(\operatorname{Ker}(v))$

$$
+\operatorname{dim} E-\operatorname{dim}(\operatorname{Im}(v))+\operatorname{dim}(\operatorname{Im}(u) \cap \operatorname{Ker}(v)) .
$$

Hence 


$$
\begin{aligned}
\operatorname{cod}(\operatorname{Im}(v \circ u)) & =\operatorname{cod}(\operatorname{Im}(u))-\operatorname{dim}(\operatorname{Ker}(v)) \\
& +\operatorname{cod}(\operatorname{Im}(v))+\operatorname{dim}(\operatorname{Im}(u) \cap \operatorname{Ker}(v)) .
\end{aligned}
$$

By subtracting (2) from (1), we have the logarithmic property:

$$
d(v \circ u)=d(v)+d(u) \text {. }
$$

\section{References}

[1] M. Audin: Sur les équations linéaires dans un espace vectoriel. Alger, Mathématique, 4, 5-75 (1957).

[2] A. Deprit: Contribution à l'étude de l'Algèbre des applications linéaires continues d'un espace localement convexe séparé. Acad. Roy. Belgique, Cl. Sci. Mém., 31, 1-170 (1959). 